\title{
Evaluation of Scalable Proxy Mobile IPv6 in Wireless Mesh Networks
}

\author{
Huu-Nghia Nguyen, Christian Bonnet \\ Mobile Communications Department \\ Eurecom \\ \{name.surname\}@eurecom.fr
}

\begin{abstract}
This paper evaluates our work of Scalable Proxy Mobile IPv6 (SPMIPv6) in a Wireless Mesh Network (WMN) which is a promising solution for ubiquitous Internet access and a wide range of applications. The aspects of scalability and signaling cost are evaluated respectively: the scalability is evaluated by numerical analysis while the signaling cost with respect to delay is evaluated by a virtualization-based method. It is shown that the SPMIPv6 extension can scale the network better than the base Proxy Mobile IPv6 (PMIPv6) protocol. The quantitative results are provided to complete our previous qualitative results and to validate the application of SPMIPv6 for WMN.
\end{abstract}

\section{Introduction}

In our previous work [1], Proxy Mobile IPv6 (PMIPv6) is extended to support the scalability in heterogeneous wireless networks. The framework, named Scalable Proxy Mobile IPv6 (SPMIPv6), inherits the features of PMIPv6 which can support mobility to Mobile Nodes (MNs) having unmodified IPv6 stack and allow reducing the flooding signaling traffic during the registration process and during the dynamic route discovery process. Moreover, thanks to a cluster-based architecture, operators are able to horizontally extend the coverage of the access network so that mobile users can be always connected.

In this paper, we evaluate the SPMIPv6 framework in the scope of Wireless Mesh Network (WMN), which has been considered as a promising solution for ubiquitous Internet access and a wide range of applications thanks to the ability of self-organizing and self-configuring [2]. The cluster-based architecture in SPMIPv6 allows for hierarchical routing in WMN by grouping network nodes into clusters that could minimize the updating overhead for topology changes.
The scalability and the signaling cost aspects are evaluated. Numerical analysis is used to evaluate the scalability with consideration of the WMN size, Mobile Node (MN) density, and average mobile speed. As regards the signaling cost, we consider a virtualization-based approach using User-mode Linux and Ns2-Emultion to evaluate the signaling cost in terms of delay.

The paper is organized as follows: Section 2 provides a background on PMIPv6 and our SPMIPv6 framework. Section 3 provides a numerical analysis on the scalability of SPMIPv6 in WMN. Section 4 evaluates the signaling cost (in terms of delay) in a virtualization-based experimental testbed using Usermode Linux and Ns-2 Emulation. Finally, section 5 concludes the paper and outlines our future research.

\section{Background}

\subsection{Proxy Mobile IPv6}

PMIPv6 [3] is designed to provide network-based localized mobility management to MNs having standard IPv6 stack which does not support IP mobility. The principal functional entities of PMIPv6 are the Mobile Access Gateway (MAG) and the Local Mobility Anchor (LMA). The main role of MAG is to detect MN's movements and initiate mobility-related signaling with the LMA on behalf of the MN. The serving network assigns a unique home network prefix to each $\mathrm{MN}$, and conceptually this prefix always follows the MN wherever it moves within a PMIPv6 domain. Therefore, the MN can keep the IP address unchanged and ensure the session continuity while moving within the PMIPv6 domain.

The base PMIPv6 protocol does not consider the route optimization for communication between MNs in the same PMIPv6 domain. Besides, a centralized LMA represents a single point of failure in a large scale 
network. If the LMA crashes for some reason, the mobility service in the whole domain is disrupted.

\subsection{Scalable Proxy Mobile IPv6 for WMN}

2.2.1. Cluster-based Architecture. The cluster-based architecture can be considered as a special architecture for hierarchical routing in WMN by grouping network nodes into clusters (see Figure 1). Each cluster, containing a Cluster Head $(\mathrm{CH})$, has complete knowledge about group membership and link state information in the cluster. The $\mathrm{CH}$ is often elected in the cluster formation process. The other nodes within a cluster, called Access Routers (ARs) in this paper, control heterogeneous radio access technologies and provide access to MNs. A relay router connects two adjacent clusters. All nodes in the backhaul are interconnected. The $\mathrm{MN}$ therefore can communicate with other mobile Correspondent Nodes (CNs) through ARs as well as with $\mathrm{CNs}$ on the Internet through CHs. When the node density is high, this architecture tend to achieve much better performance because of less overhead, shorter average routing path, and quicker set-up procedure of routing path.

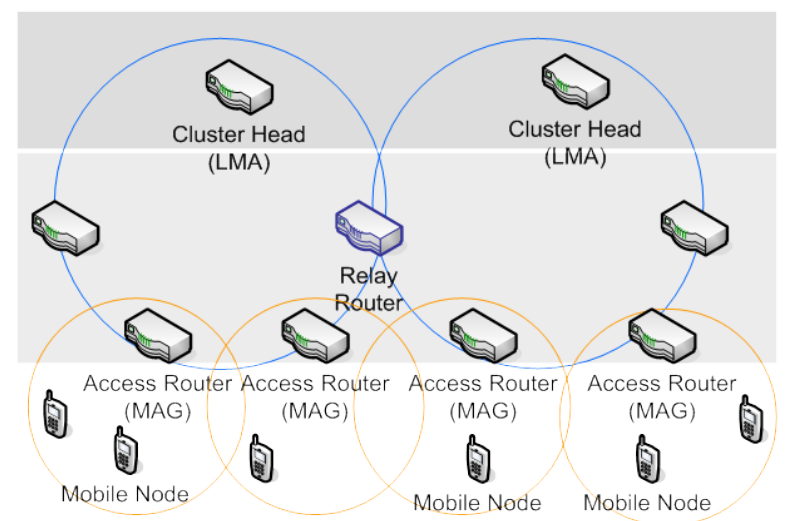

Figure 1. Cluster-based architecture

The base PMIPv6 provides a natural solution for communication between an $\mathrm{MN}$ and a $\mathrm{CN}$ located outside the PMIPv6 domain. It is also efficient for intra-cluster communication and intra-cluster mobility. Thanks to the use of IP tunneling between the LMA and the MAG, PMIPv6 hides away the topology within the backhaul link from the MNs. However for intercluster communication, when the $\mathrm{MN}$ and the $\mathrm{CN}$ belong to different clusters in the same PMIPv6 domain, one fundamental issue is that of locating the serving MAG or the serving LMA of the CN. As regards inter-cluster mobility, when the $\mathrm{MN}$ moves from one cluster to a new cluster, it is necessary to activate the Location Deregistration procedure in the old cluster to maintain up-to-date routing information. We need to extend the protocol to solve the following fundamental issues: (i) detecting the communication establishment, (ii) locating the serving entities of the $\mathrm{CN}$ and (iii) maintaining up-to-date routing information.

2.2.2. Detecting Communication Establishment. We define the communication in this work as the exchange of traffic between two nodes. By inspecting Internet Control Message Protocol (ICMP) traffic or data traffic, this process determines the scope of communications, i.e. intra-communication or intercommunication, and provides triggers for route setup in case of inter-communication or route optimization.

2.2.3. Locating the Serving Entities. Let $M A G_{M N}$ and $\mathrm{LMA}_{\mathrm{MN}}$ denote the serving MAG and the serving LMA of the $\mathrm{MN}$ respectively. Also let $\mathrm{MAG}_{\mathrm{CN}}$ and LMA $_{\mathrm{CN}}$ denote the serving MAG and the serving LMA of the $\mathrm{CN}$ respectively. When establishing the communication between an $\mathrm{MN}$ and a $\mathrm{CN}$ belonging to different clusters, $\mathrm{LMA}_{\mathrm{MN}}$ needs to know $\mathrm{LMA}_{\mathrm{CN}}$ and $\mathrm{MAG}_{\mathrm{CN}}$. This location issue is expressed as the problem of mapping a $\mathrm{CN}$ address into its serving MAG address and its serving LMA address. To solve this location issue, we propose a new couple of messages: Proxy Binding Request (PBReq) and Proxy Binding Response (PBRes). Five new options are also defined and can also be used for route optimization which is out of scope of this paper.

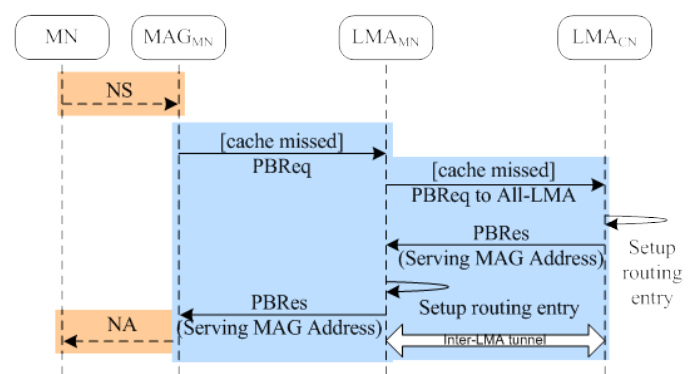

\section{Figure 2. Inter-clusters communication establishment}

The inter-clusters communication establishment is illustrated in Figure 2. Once the $\mathrm{MAG}_{\mathrm{MN}}$ detects a new communication, $\mathrm{MAG}_{\mathrm{MN}}$ looks up the $\mathrm{CN}$ address in its binding cache. If no information is found for that $\mathrm{CN}$ belonging to the same PMIPv6 domain, i.e. cache missed, the $\mathrm{MAG}_{\mathrm{MN}}$ assumes that the $\mathrm{CN}$ is away from its home link and sends a PBReq message to the $\mathrm{LMA}_{\mathrm{MN}}$. If the $\mathrm{LMA}_{\mathrm{MN}}$ does not have any information about the target, it must send a PBReq to All-LMA multicast address. The $\mathrm{LMA}_{\mathrm{CN}}$ will reply with a PBRes 
carrying at least the $\mathrm{MAG}_{\mathrm{CN}}$ address which can be used for route optimization. The $\mathrm{LMA}_{\mathrm{MN}}$ and $\mathrm{LMA}_{\mathrm{CN}}$ can setup a routing entry pointing to the $\mathrm{LMA}_{\mathrm{MN}}-\mathrm{LMA}_{\mathrm{CN}}$ bidirectional tunnel. As a result, a default path traversing LMAs is set up for the communication between the $\mathrm{MN}$ and the $\mathrm{CN}$. The $\mathrm{LMA}_{\mathrm{MN}}$ then will reply with a PBRes to the $\mathrm{MAG}_{\mathrm{MN}}$ to inform the $\mathrm{MAG}_{\mathrm{MN}}$ about the $\mathrm{MAG}_{\mathrm{CN}}$ address. Later, the traffic from $\mathrm{MN}$ to $\mathrm{CN}$ can be delivered through a chain of bidirectional tunnels: $\mathrm{MAG}_{\mathrm{MN}}-\mathrm{LMA}_{\mathrm{MN}}$, $\mathrm{LMA}_{\mathrm{MN}}{ }^{-}$ $\mathrm{LMA}_{\mathrm{CN}}$ and $\mathrm{MAG}_{\mathrm{CN}}-\mathrm{LMA}_{\mathrm{CN}}$. When shared prefix scheme is used on access links, the $M_{A A G}$ must perform Proxy ARP for the CN.

As regards the reverse path from the $\mathrm{CN}$ to the $\mathrm{MN}$, it will be set up in the same manner. To speed up reverse path setup, a caching mechanism is used. This cache is also used to maintain inter-cluster paths.

2.2.4. Maintaining up-to-date routing information. When the MN moves from one cluster to a new cluster, the old LMA may not be aware about the changes, the new LMA can advertise a PBRes message to All-LMA multicast address. This message helps the old LMA to activate the Location Deregistration procedure if necessary, and helps other LMAs to maintain up-todate routing information to keep on-going session.

\section{Numerical analysis on the scalability}

We use mathematical model, inspired from [4], to investigate the scalability of PMIPv6 and SPMIPv6 in a WMN with consideration of the WMN size, MN density, and average mobile speed. We wish to state that the analysis is approximate and contains simplifying assumptions made for the sake of analytical tractability. However it can still provide a macroscopic view of the scalability.

\subsection{Assumptions}

We consider the WMN with hexagonal cell structure, assuming that each cell is served by an AR. The coverage area of a cluster is also hexagon shaped. For PMIPv6, one LMA can serve the whole WMN, but once SPMIPv6 is introduced, each LMA resides at one $\mathrm{CH}$ and serves only its cluster. Each LMA has a limited capacity beyond which the system performance degrades exponentially. Therefore to avoid the overloaded situation, LMAs use access control policy to reject handover attempts causing overload. The structure of a cluster is shown in Figure 3. Let $K$ denote the size of a cluster, so the number of ARs in a cluster is

$$
N_{K}=3 K(K-1)+1
$$

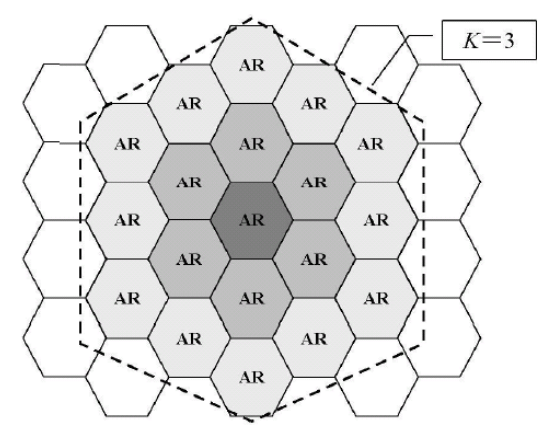

Figure 3. Structure of a cluster

The perimeter of a AR cell is $l$, so the perimeter of a cluster denoted as $L_{K}$ is

$$
L_{K}=(2 K-1) l
$$

The fluid flow mobility model is widely used to analyze subnets boundary crossing problems, such as handover. Thus, we use this model to investigate the scalability of the WMN while taking into account the mobility of MNs. In fluid flow mobility model, MNs are moving at an average velocity of $\mathbb{E}[v]$ in uniformly distributed directions over $[0,2 \pi]$ and also uniformly populated with a density $\rho$.

The random variable $T_{d w e l l}$, the dwell time of mobile users in a cell, has exponential distribution with mean $T_{d w e l l}=\frac{1}{\mu_{d w e l l}}$ is assumed. The model assumes a uniform density of users throughout the area and also assumes that a user is equally likely to move in any direction with respect to the cell boundary. For twodimensional models, we know that the average outgoing rate of mobile users is given by

$$
\mu_{d w e l l}=\frac{\mathbb{E}[v] l}{\pi A}
$$

\subsection{Per-cell Handover Rate}

We assume that active users are always in connected state. We know that the rate of per-cell handover $r_{h}$ is given by

$$
r_{h}=\frac{\rho \mathbb{E}[v] l}{\pi}
$$

\subsection{Handover failure probability}

Let $S=S_{0}$ be the capacity of one LMA. This means that one LMA can serve, in maximum, $S_{0}$ mobile users. Beyond this capacity, the system performance degrades exponentially. Given $S_{0}$, we can always configure a right value of cluster size $K$ such that an LMA is never overloaded.

To scale the WMN in a horizontal manner, we increase the value of $N_{C}$ parameter. As regards vertical 
scalability, we can replace the LMA by a more power LMA which has the capacity $S=k S_{0}(k>1)$.

Let $P_{B}$ to be fraction of handover attempts fails due to access control policy. We define the state $E_{j}$ of the LMA such that a total of $j$ users are served successfully. Let $P_{j}$ represent the steady-state probability that the LMA is in the $E_{j}$ state, the probability can be determined in the usual way for birth-death processes. On a geographic area equivalent to that of $N_{C}$ cluster, Let $\mathrm{N}_{\mathrm{MAG}}$ represent the number of MAGs that one LMA covers. With PMIPv6, a single LMA is used to control the whole WMN, then

$$
N_{M A G}=N_{C} N_{K}
$$

Otherwise with SPMIPv6, we use multiple LMAs to control the WMN; each LMA resides at the $\mathrm{CH}$ and controls only one cluster, then

$$
N_{M A G}=N_{K}
$$

The intensity $\alpha$ and the blocking probability $P_{B}$ are:

$$
\begin{gathered}
\alpha=\frac{N_{M A G} r_{h}}{\mu_{d w e l l}}=N_{M A G} \rho A \\
P_{B}=P_{S}=\frac{\frac{\alpha^{S}}{S !}}{\sum_{i=0}^{S} \frac{\alpha^{i}}{i !}}
\end{gathered}
$$

\subsection{Numerical Results}

Numerical results are obtained using MATLAB $\mathbb{R}$ R2006b. We realized 500 simulations. For each simulation, we model the increasing of the WMN's geographic area by increasing the $N_{C}$ parameter. We model the upgrade of an LMA by increasing $k$ parameter in a limited range from 1 to 5 because the capacity might be unlimited in theory but is limited in the practice. Other system parameters for numerical analysis are taken randomly in the range shown in Table 1.

Table 1. System parameter for numerical analysis

\begin{tabular}{cll}
\hline \hline Parameter & Values & Unit \\
\hline$S_{0}$ & 250 & nodes \\
$N_{C}$ & $\{1 . .20\}$ & clusters \\
$K$ & $\{2 . .3\}$ & \\
$l$ & $\{200 \pi . .400 \pi\}$ & $\mathrm{m}$ \\
$\mathbb{E}[v]$ & $\{1 . .5\}$ & $\mathrm{m} / \mathrm{s}$ \\
$\rho$ & $\{0.0001 . .0 .0002\}$ & nodes $/ \mathrm{m}^{2}$ \\
$k$ & $\{1 . .5\}$ & \\
$A$ & $\sqrt{3} l^{2}$ & $\mathrm{~m}^{2}$ \\
& $\frac{}{24}$ & \\
\hline
\end{tabular}

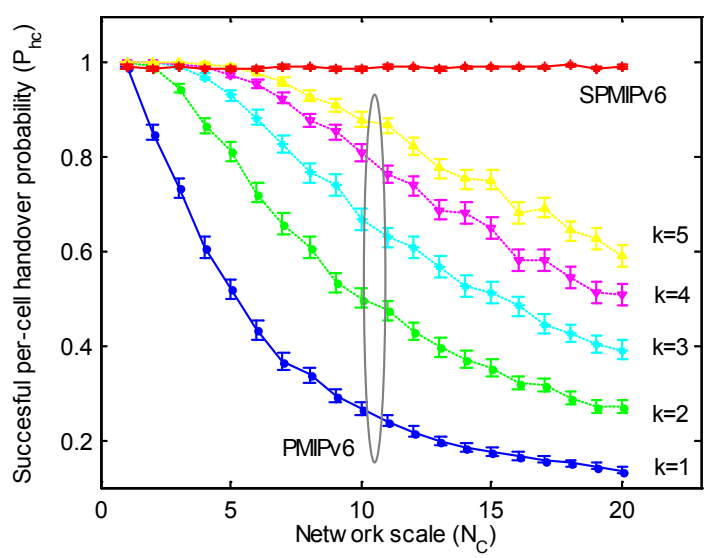

Figure 4. Successful per-cell handover probability

In this part, we analyze the scalability feature of SPMIPv6 with respect to successful per-cell handover probability and successful per-cell handover rate. Figure 4 shows the probability of successful per-cell handover in SPMIPv6 and different cases of PMIPv6. Let $P_{h c}$ denote the probability that a per-cell handover completes successfully. We observe that, when the network is extended horizontally (by increasing $N_{C}$ parameter), $P_{h c}$ with only one LMA decreases dramatically; while $P_{h c}$ with multiple LMAs keeps almost stable. The figure shows five different values of $k$ corresponding to five different capacity of the centralized LMA. This means that we can vertically scale the WMN using a new LMA with larger capacity (by increasing $k$ parameter). However the capacity might be unlimited in theory but is limited in the practice. Besides it is always costly to replace a centralized LMA by a new one with larger capacity.

With the fluid flow mobility model, the rate at which per-cell handovers complete is:

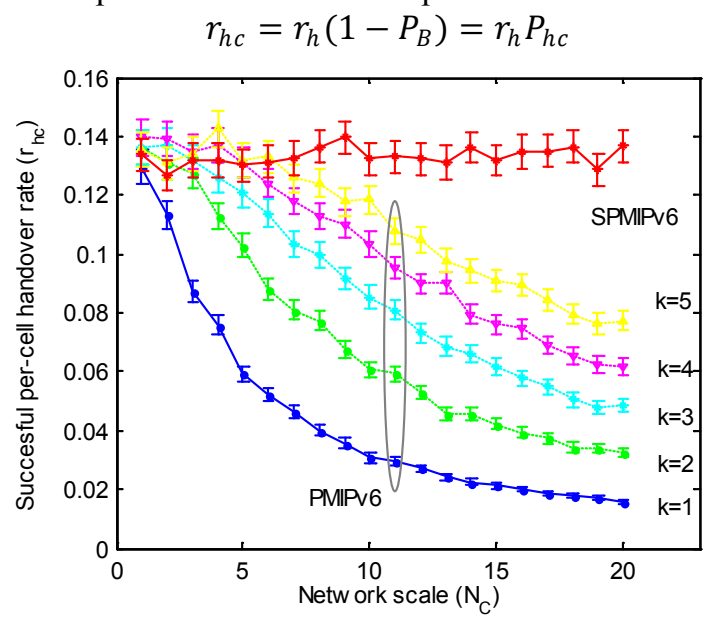

Figure 5. Successful per-cell handover rate 
Figure 5 shows the successful per-cell handover rate when the network scale varies. Note that this rate depends on the scale of the network and also on the average velocity of MNs. When the network scale increases gradually, SPMIPv6 out performs PMIPv6 as no handover is rejected.

\section{Signaling Cost Evaluation}

\subsection{Virtual IPv6 Wireless Mesh Network}

To evaluate the signaling cost of the SPMIPv6 in WMN, we use a combination of User-mode Linux (UML) [5] and Ns-2 Emulation [6], with the scope of being as close as possible to real experimentation results and to easily migrate to the real testbed in the near future. The topology is generated by the Virtual Network User-mode Linux (VNUML) [7].

The virtual WMN (see Figure 6) is composed of two clusters under the control of $\mathrm{CH} 1$ and $\mathrm{CH} 2$, and three routers AR1, AR2 and AR3. LMA functionality runs on $\mathrm{CHs}$ while MAG functionality runs on ARs. AR1 and AR2 are under the control of CH1. AR3 is under the control of $\mathrm{CH} 2$. $\mathrm{CH} 1$ and $\mathrm{CH} 2$ are interconnected.

MN1 and MN2 do not have any specific software for mobility management and can be initially attached to any ARs. For simplification, the access links use IEEE 802.11 access technology. MNs' addresses are auto-configured thanks to IPv6 Stateless Address Auto Configuration. We assume that there is no IPv6 address conflict, and use a shared-prefix model with a shared prefix of $2001: 1:: / 64$. The three site-scope prefixes FEC0:1000::/64, FEC0:2000::/64 and FEC0:3000::/64 are used for enhanced network-based movement detection procedure [2]. Three ARs are configured with Router Advertisement daemons (RADVD) which broadcast Router Advertisements (RAs) on their eth0 interface. RAs contain two prefixes and are periodically sent every $100 \mathrm{~ms}$.

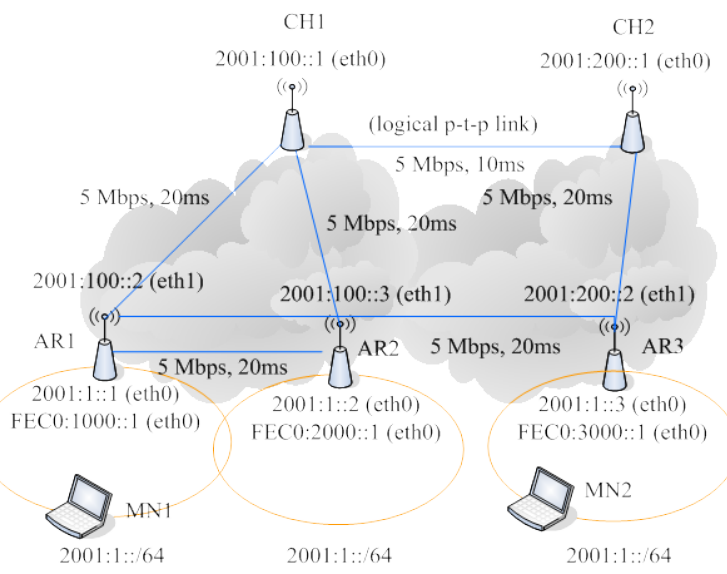

Figure 6. Virtual Wireless Mesh Network
Ns2-Emultion is used to emulate the wireless links between nodes. The logical connectivity between entities in the mesh backhaul is represented by Ns2 point-to-point links which are characterized by bandwidth and delay. This allows us to impose specific delay in the transmission of messages between entities to produce emulation results that are closest to real experimentation results.

\subsection{Signaling cost in terms of delay}

To scale the network gradually and horizontally with multiple clusters, extra signaling is needed. This introduces extra delay during the communication setup phase. To measure the extra delay caused by the signaling mechanism, we use the ping6 tool and measure the RTT of the first packet in two scenarios: (i) pre-established route without signaling and (ii) ondemand route with signaling. Let $r_{l}$ be the random variable representing the RTT of the first ping packet with signaling, and $r_{2}$ be the random variable representing the RTT of the first ping packet with preestablished route without signaling. In both cases, we include also the time of Neighbor Unreachability Detection (NUD) procedure between MNs and their serving MAGs. The average signaling cost in terms of delay can be calculated as mean $\left(r_{1}\right)$-mean $\left(r_{2}\right)$.

4.2.1. Intra-clusters Communication. This scenario considers the communication of two MNs attached to two different ARs inside the same cluster: MN1 is attached to AR1 while MN2 is attached to AR2. Both AR1 and AR2 are under the control of CH1. Once registered with the Location Registration process, the two MNs can communicate with each other through the AR1-CH1-AR2 path using two IPv6 tunnels. The scenario is repeated 50 times; 50 samples of $r_{l}$ and 50 samples of $r_{2}$ are captured.

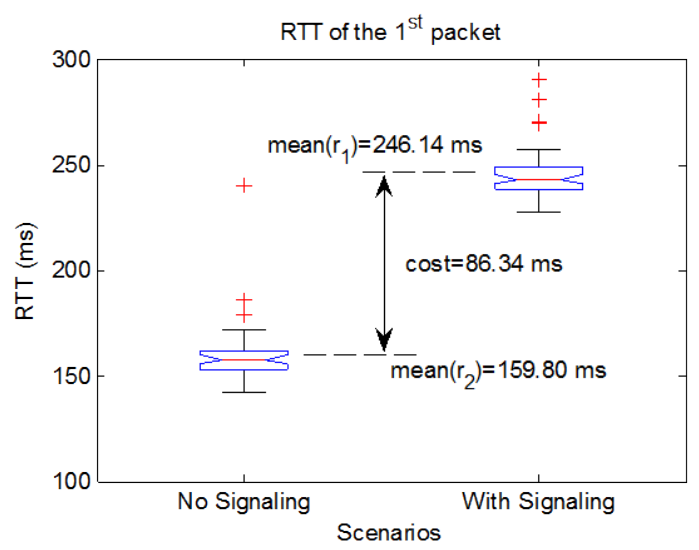

Figure 7. Signaling cost in terms of delay in intra-cluster communication 
Figure 7 shows the distribution of $r_{1}$ and $r_{2}$ in form of box-and-whisker diagram in the intra-cluster communication scenario. Box-and-whisker diagram is a convenient way of graphically depicting groups of numerical data through their five-number summaries (the lower extreme, lower quartile, median, upper quartile, and upper extreme). The average signaling cost is then calculated and depicted as the difference between the mean of $r_{1}$ and the mean of $r_{2}$. In our virtual testbed, it takes in average $86.34 \mathrm{~ms}$ for establishing a new communication. This delay depends on both the processing time at edge entities and the message exchange delay between them. In comparison with the RTT of ping packets between the two MNs, which has the average value of $90.15 \mathrm{~ms}$ over 500 samples, the extra delay for the first packet is almost the same and quite acceptable; especially as this extra delay happens only once during the communication.

4.2.2. Inter-clusters Communication. This scenario considers the communication of two MNs attached to two different ARs belonging to different clusters: MN1 is attached to AR1 under the control of $\mathrm{CH} 1$, while MN2 is attached to AR3 under the control of $\mathrm{CH} 2$. Once registered, the two MNs can communicate with each other through the AR1-CH1-CH2-AR2 path using three IPv6 tunnels. We apply the same measurement as in the section 4.2.1 to evaluate the signaling cost.

Figure 8 shows the distribution of $r_{1}$ and $r_{2}$ in form of box-and-whisker diagram. In this virtual testbed, it takes in average $138.08 \mathrm{~ms}$ for establishing a new inter-clusters communication. This delay is slightly more important than the one measured in the intracluster scenario. This is due to the presence of the additional LMA and the additional inter-LMA link which increase the overall processing time and the message exchange delay. In comparison with the RTT

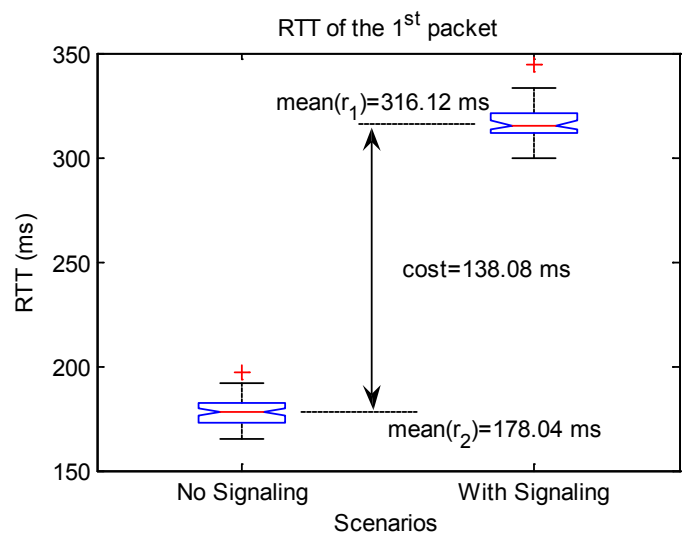

Figure 8. Signaling cost in terms of delay in inter-cluster communication of ping packets between the two MNs, which has the average value of $111.35 \mathrm{~ms}$ over 500 samples, the extra delay for the first packet is still quite acceptable; especially as this extra delay happens only once during the communication.

\section{Conclusion}

We have evaluated the scalability our SPMIPv6 framework in a WMN context. A mathematical model has been used to investigate the scalability of the framework with consideration of the WMN size, MN density, and average mobile speed. The metrics, used to reflect the scalability, are the probability of successful per-cell handover and the successful per-cell handover rate. These metrics have been calculated and compared between PMIPv6 and SPMIPv6. Numerical results shows that SPMIPv6 provides a mechanism for inter LMAs interactions which can horizontally and gradually scale the WMN. This approach is less expensive than replacing the centralized LMA and avoids the single point of failure problem in PMIPv6. The SPMIPv6 signaling cost has also been evaluated in a virtual WMN. The cost is expressed in terms of delay caused by the extra signaling in the beginning of the communication. Our results show that the signaling cost is reasonable, especially as it happens only once for each communication.

\section{References}

[1] H.N. Nguyen, C. Bonnet, "Scalable proxy mobile IPv6 for heterogeneous wireless networks", International Workshop on Mobile IPv6 and Network-based Localized Mobility Management, I-Lan, Taiwan, September 2008.

[2] I. F. Akyildiz and X. Wang, "A Survey on Wireless Mesh Networks", IEEE Comm. Magazine, vol. 43, no. 9, 2005, pp. 23-30.

[3] S. Gundavelli, K. Leung, V. Devarapalli, K. Chowdhury, B. Patil, "Proxy Mobile IPv6", IETF RFC 5213, August 2008.

[4] Daehyoung, H. and S.S. Rappaport, "Traffic models and performance analysis for cellular mobile radio telephone systems with prioritized and non-prioritized handoff procedures", IEEE. Transactions on Vehicular Technology, vol VT-35, no. 3, 1986, pp.77-92

[5] User Mode Linux Home Page, http://user-modelinux.sourceforge.net

[6] Daniel Mahrenholz and Svilen Ivanov, "Real-Time Network Emulation with ns-2," Proceedings of The 8-th IEEE International Symposium on Distributed Simulation and Real Time Applications, Budapest Hungary, October 21-23, 2004.

[7] Virtual Network User Mode Linux Home page http://www.dit.upm.es/vnumlwiki/index.php/Main_Page 